20

\title{
Спектральный анализ костной ткани крыс при длительном антиортостатическом вывешивании и введении аллогенного гидроксиапатита
}

\author{
(C Е.В. Тимченко ${ }^{1}$, П.Е. Тимченко ${ }^{1, \uparrow, ~ Е . В . ~ П и с а р е в а ~}{ }^{1}$, М.Ю. Власов ${ }^{2}$, Л.Т. Волова ${ }^{2}$, О.О. Фролов ${ }^{1}$, \\ Я.В. Федорова ${ }^{1}$, Г.П. Тихомирова ${ }^{1}$, Д.А. Романова ${ }^{1}$, М.А. Даниэль ${ }^{1}$ \\ ${ }^{1}$ Самарский национальный исследовательский университет имени академика С.П. Королева (Самарский университет), \\ 443086 Самара, Россия \\ ${ }^{2}$ Самарский государственный медицинский университет, институт экспериментальной медицины и биотехнологий, \\ 443099 Самара, Россия \\ Te-mail: laser-optics.timchenko@mail.ru
}

Поступила в редакцию 21.01.2020 г.

В окончательной редакции 02.03.2020 г.

Принята к публикации 28.03.2020 г.

Исследована костная ткань крыс при моделировании остеорезорбции в условиях микрогравитации. Были исследованы образцы костей крыс самок и самцов с остеопорозом при лечении гидроксиапатитом с помощью метода спектроскопии комбинационного рассеяния. Установлены спектральные различия между исследуемыми образцами (контрольные образцы, образцы с моделью остеопороза, образцы с моделью остеопороза при лечении гидроксиапатитом).

Ключевые слова: спектроскопия комбинационного рассеяния, спектр, микрогравитация, остеопороз, гидроксиапатит.

DOI: $10.21883 /$ OS.2020.07.49571.75-20

\section{Введение}

В настоящее время лазеры находят широкое применение во многих областях [1-3]. Микрогравитация, испытываемая космонавтами во время космических полетов, вызывает ускоренную потерю костной массы, происходит нарушение минерального обмена костной ткани, что приводит к развитию остеопороза. Процесс адаптации костной ткани к условиям микрогравитации выражается в её ремоделировании [4], сопровождается изменением кинетики кальция [5] и отклонением уровня биохимических маркеров метаболизма [6]. Их динамика может дать ценную информацию о балансе процессов резорбции и новообразования кости после космического полета и в восстановительном периоде. Исследование обменных процессов костной ткани после космических полетов проводилось с самого начала освоения человеком космоса, но только относительно недавно по мере расширения спектра биомаркеров и с появлением новых методов их изучения появились возможности проводить анализ архивных образцов. Однако к настоящему времени полученные по ним данные немногочисленны $[7,8]$. Развитие метаболических изменений в скелетно-мышечной системе может быть связано со снижением нагрузки на опорно-двигательный аппарат [9]. Эти процессы в длительном космическом полёте проявляются повышением белкового катаболизма, отрицательным балансом азота и увеличением экскреции некоторых аминокислот и их метаболитов, таких как креатинин, саркозин, 3метилгистидин, гидроксипролин, с одновременным из- менением активности ряда гормонов [10]. Обнаружено также снижение способности к депонированию калия в мышечной ткани и кальция в костной ткани, что проявляется отрицательным балансом кальция.

У космонавтов в условиях космического полета увеличивается выведение кальция и $C$-концевых телопептидов коллагена I типа с мочой, а в сыворотке крови снижается содержание таких маркеров формирования костной ткани, как остеокальцин и $C$-терминальный пропептидпроколлагена I типа [11]. Предполагается, что подобные изменения связаны с угнетением функции остеобластов с одновременным усилением остеокластической активности. Считается, что ключевую роль в регуляции функции клеток костной ткани играет экспрессия некоторых белков, в частности склеростина, являющегося ингибитором формирования костной ткани в том числе и в условиях разгрузки конечностей [12]. Таким образом, модель ортостатической разгрузки задних конечностей грызунов позволяет оценить в эксперименте последствия воздействия микрогравитации на обмен костной ткани.

Ранее были изучены остеопротективные свойства гидроксиапатита на глюкокортикоидной модели у крыс. Установлено, что применение аллогенного гидроксиапатита способствовало снижению резорбции костной ткани и нормализации процессов метаболизма [13]. В связи с этим представляет интерес изучение показателей обмена костной ткани в условиях антиортостатической разгрузки как изолированно, так и при комбинировании с введением гидроксиапатита и глюкокортикоидов, по- 
скольку известно, что экзогенное введение глюкокортикоидов является моделированием состояния остеопороза [14]. Исходя из вышеизложенного актуальной задачей является анализ костной ткани при моделировании остеорезорбции в условиях микрогравитации и поиск новых способов профилактики и лечения остеопороза с помощью биосовместимых аллогенных материалов, одним из которых является гидроксиапатит.

При изучении остеопороза особое внимание уделяется содержанию костной массы, чье изменение может привести к снижению прочности кости. С помощью неинвазивного метода денситометрии можно определить минеральную плотность костной ткани. Рентгеновская костная денситометрия в настоящее время - самый распространенный вид денситометрии и основной способ диагностики заболеваний при нарушении минерализации кости [15]. Одним из недостатков метода является системная ошибка из-за неравномерности содержания жира в жиросодержащих тканях (жир и желтый костный мозг) различных участков тела и костей. На результаты исследований также могут влиять изменения состава тела, т.е. жировой и тощей массы вокруг области сканирования.

Методы спектроскопии считаются сегодня наиболее перспективными в современной неинвазивной оптической диагностике. Данный метод применяется в качестве инструмента для мониторинга динамики процессов, происходящих в тканях, дает уникальные возможности изучения возбужденных состояний молекул, фотохимических реакций, динамики быстрых молекулярных процессов, структуры и свойств сложных химических и биологических объектов [16].

Целью работы является применение метода спектроскопии комбинационного рассеяния (КР) для спектральной оценки костной ткани при моделировании остеорезорбции в условиях микрогравитации.

\section{Материалы и методы исследований}

Эксперимент проводили на 60 (30 самок и 30 самцов) взрослых беспородных лабораторных крысах весом 200-280g, которые были поделены на 5 групп (по 6 животных разного пола в группе) в соответствии со следующей схемой исследования. Животные первой группы подвергались воздействию микрогравитации в течение 28 суток посредством антиортостатического вывешивания лабораторных животных по методу Новикова-Ильина [17]. Во вторую группу входили животные, подвергавшиеся воздействию микрогравитации и глюкокортикоидов в течение 28 суток, внутрибрюшинные инъекции препарата гидрокортизон „Гедеон Рихтер“ в дозе $40 \mathrm{mg} / \mathrm{kg}$. Третья группа аналогична второй с дополнительным введением гидроксиапатита (ГАП) в дозе $100 \mathrm{mg} / \mathrm{kg}$. Четвертая группа аналогична первой с дополнительным введением ГАП в дозе $100 \mathrm{mg} / \mathrm{kg}$. Пятая группа - интактные животные (контроль).

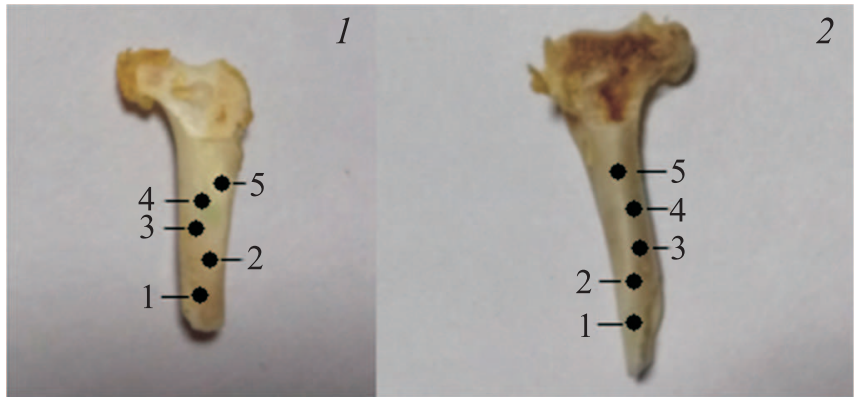

Рис. 1. Образцы кортикальных костей: 1 - самки, 2 - самцы. Точками обозначены исследуемые зоны.

В динамике животных выводили из эксперимента путем декапитации. Материалом для исследования послужили сыворотка крови и образцы бедренных, плечевых, тазовых, лопаточных и нижнечелюстных костей. В сыворотке крови определяли концентрацию концевых телопептидов (CTx, $\beta$-CrossLaps) на полуавтоматическом иммуноферментном анализаторе „StatFax 3200“ с помощью набора „Rat-laps“ (Cloud Clone, CША). Проводили морфологические исследования препаратов бедренных костей животных. Препараты окрашивали гематоксилинэозином, микроскопия осуществляли при 200-кратном увеличении на микроскопе LeicaDM4000B. Статистическую обработку проводили согласно принятой методике с использованием $t$-критерия Стьюдента, с применением программ MicrosoftExcel 2010 и SigmaPlot 12.0. При проверке статистических гипотез пороговый уровень значимости $\alpha$ составил 0.05. Проверку на нормальность распределения выборок проводили с использованием критерия Шапиро-Уилка. При сравнении двух несвязанных совокупностей и нормальном распределении применяли $t$-критерий Стьюдента.

Определение микротвердости костей проводили по Виккерсу на микротвердомере ПМТ-3, алмазная пирамидка которого вдавливалась при нагрузке $100 \mathrm{~g}$. Значения микротвердости вычислялись по формуле

$$
H \mu=(1854.4 m) /\left(d \cdot 10^{-3}\right)^{2},
$$

где $m=100 \mathrm{~g}, d-$ среднее значение диагоналей отпечатка, $\mathrm{mm}$. Измерения диагоналей отпечатков производились с помощью медицинского микроскопа БИОМЕД MMP-2, оснащенного цифровой камерой ToupCamUCMOS05100KPA.

Проводили исследование поверхности бедренных костей с помощью метода спектроскопии комбинационного рассеяния (СКР). В образцах исследовали кортикальную костную ткань самцов и самок крыс в пяти разных точках (рис. 1). Образцы исследовали с помощью стенда, реализующего метод СКР. Стенд, изображенный на рис. 2, состоит из высокоразрешающего цифрового спектрометра Shamrocksr-303i, встроенной охлаждаемой камеры DV420A-OE (со спектральным диапазоном 200-1200 nm), волоконно-оптического зонда RPB-785 


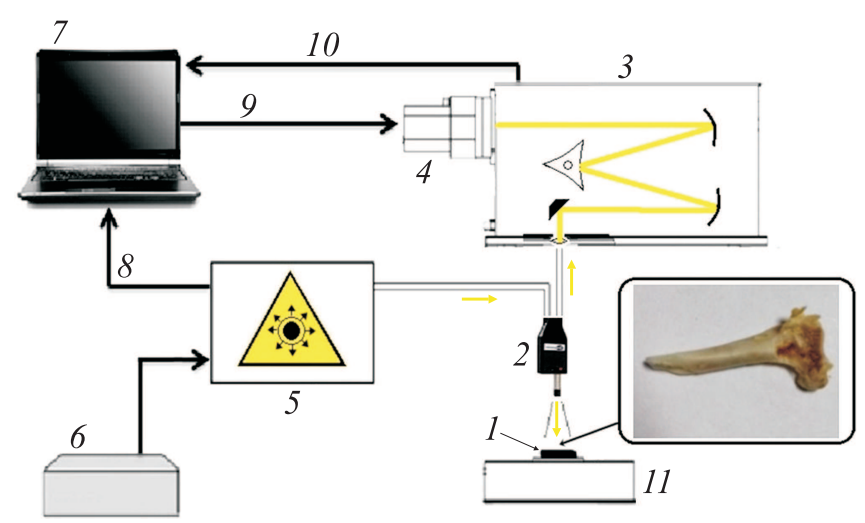

Рис. 2. Экспериментальный стенд: 1 - исследуемый объект; 2 - рамановский пробник RPB-785; 3 - спектрометр Shamrock sr-303i; 4 - встроенная охлаждаемая камера DV420A-OE; 5 - лазерный модуль LuxxMasterRamanBoxx 785.0RB-04; 6 - источник питания лазерного модуля; 7 компьютер; 8-10 - информационные электрические кабели; 11 - координатный стол.

для СКР, совмещённого с лазерным модулем LuxxMaster LML-785-0RB-04 с длиной волны лазерного излучения $785 \mathrm{~nm}$ и шириной линии $<0.1 \mathrm{~nm}[18,19]$. Обработка полученных спектров была выполнена в программе WolframMathematica 9. Исследуемый спектр при обработке очищали от шумов сглаживающим медианным фильтром (5 точек), определяли на выбранном интервале $700-2200 \mathrm{~cm}^{-1}$ при помощи итерационного алгоритма аппроксимирующую линию (полином пятой степени) автофлуоресцентной составляющей, а затем вычитали эту составляющую, в результате получали выделенный спектр [20,21].

\section{Результаты исследований}

На рис. 3 представлены усредненные спектры КР, полученные при исследовании плечевых костей самок и самцов крыс с моделью остеопороза и с лечением ГАП. Расшифровка основных линий приведена в табл. 1. Как видно из рис. 3 , основные спектральные различия между моделью остеопороза и моделью остеопороза с лечением ГАП наблюдаются на линиях $855 \mathrm{~cm}^{-1}$ (гидроксипролин $), 956 \mathrm{~cm}^{-1}\left(\mathrm{PO}_{4}^{3-}\right.$, симметричные валентные колебания фосфата), $1069 \mathrm{~cm}^{-1}$ (карбонат, валентные колебания $\left.\mathrm{CO}_{3}^{2}\right)$. Увеличение содержания гидроксипролина (соответствующее линии $855 \mathrm{~cm}^{-1}$ ), входящего в состав белка коллагена и связанного с пептидами костного матрикса, в контрольных образцах и образцах с лечением ГАП может свидетельствовать о снижении резорбтивных процессов в кости [22]. Минералы костной ткани, соответствующие линиям 956 и $1069 \mathrm{~cm}^{-1}$, отвечают за качество и прочность кости [19]. Характерная карбонатная полоса $1069 \mathrm{~cm}^{-1}$ в спектре КР также указывает на фосфатные позиции в апатитной решетке, которые склонны к ионному замещению, известному
Таблица 1. Расшифровка основных линий КР

\begin{tabular}{|c|c|}
\hline$k, \mathrm{~cm}^{-1}$ & Вещество, колебание \\
\hline 815 & $\begin{array}{c}\mathrm{v}(\mathrm{C}-\mathrm{O}-\mathrm{O}) ; \mathrm{v}(\mathrm{C}-\mathrm{C}) \text { of backbone } \\
\text { skeletal vibrations }[26]\end{array}$ \\
\hline 855 & Hydroxyproline v $(\mathrm{C}-\mathrm{C})$ stretch $[27]$ \\
\hline 956 & v1 $\mathrm{P}-\mathrm{O}$ symmetric stretch $\left(\mathrm{PO}_{4}^{3-}\right)[28]$ \\
\hline 1004 & Phenlyalanine $[29,30]$ \\
\hline 1069 & $\mathrm{C}-\mathrm{O}$ in plane stretch $\left(\mathrm{CO}_{3}^{2}\right.$ v1 $)[27,28]$ \\
\hline 1165 & $\mathrm{v}(\mathrm{C}-\mathrm{O}-\mathrm{C})$ symmetric stretch \\
\hline 1245 & Amide III $(\mathrm{C}-\mathrm{N}-\mathrm{H}$ stretch $)[27,31]$ \\
\hline 1270 & Amide III, ( $\alpha$-helix) $(\mathrm{C}-\mathrm{N}-\mathrm{H}$ растяжение) [31] \\
\hline 1340 & Amide III, $\left(\mathrm{CH}_{2}\right.$ deformation) $(\alpha$-helix $)[26]$ \\
\hline 1420 & Proteins, Lipids $(\mathrm{C}-\mathrm{N}$ bend $)[32,33]$ \\
\hline 1425 & $\mathrm{C}-\mathrm{H}$ bending vibration $(\delta \mathrm{C}-\mathrm{H})[26]$ \\
\hline 1445 & $\begin{array}{c}\mathrm{CH}_{2} \text { изгиб и ножничные режимы } \\
\text { коллагена и фосфолипидов }\end{array}$ \\
\hline 1560 & Amide II $(\mathrm{C}-\mathrm{N}$ bend $)[33]$ \\
\hline 1665 & Amide I (random coils) [34] \\
\hline 1739 & Phospholipids $(\mathrm{C}=\mathrm{O})$ \\
\hline
\end{tabular}

как карбонатное замещение $B$-типа [23]. Жесткость и модуль изгиба кости существенно зависят от степени минерализации, минеральной кристалличности и замещения карбоната $B$-типа. Усиление замещения карбоната $B$-типа при остеопорозе приводит к увеличению хрупкости костей $[24,25]$.

Амид III и амид I, представленные основными линиями на 1245,1270 и $1665 \mathrm{~cm}^{-1}$, соответственно отнесены к коллагеновым структурам, значительно не меняются при сравнении контрольных и образцов с моделью остеопороза самок и самцов.

Для более детального исследования был проведен анализ амплитуд разделенных линий, нормированных на интенсивность линии $1739 \mathrm{~cm}^{-1}$ (phospholipids) c помощью метода линейного дискриминантного анализа (LDA). Данный метод реализован в программной среде SPSSStatistics 23. Результаты анализа различий групп образцов костных тканей представлены в виде набора данных трех моделей: графики счетов (рис. 4, 6, 8) и графики наиболее информативных переменных структурной матрицы (рис. 5, 7, 9).

Анализ взаимосвязи групп объектов по признакам влияния ежедневного введения глюкокортикоидов в дозе $40 \mathrm{mg} / \mathrm{kg}$ представлен на рис. 4. Показано, что основные отличия между двумя группами образцов описывает дискриминантная функция LD-1. Выборка составляет 121 спектр КР. Положительные значения LD-1 характерны для спектров КР, полученных для образцов под влиянием глюкокортикоидов, отрицательные - для контрольной группы. Области групп имеют значительное пересечение, влияющее на процент корректно классифицированных объектов при кросс-проверке.

Распределения значений функции для групп были аппроксимированы функцией нормального распределения. Наиболее значимые различия между группами образцов 


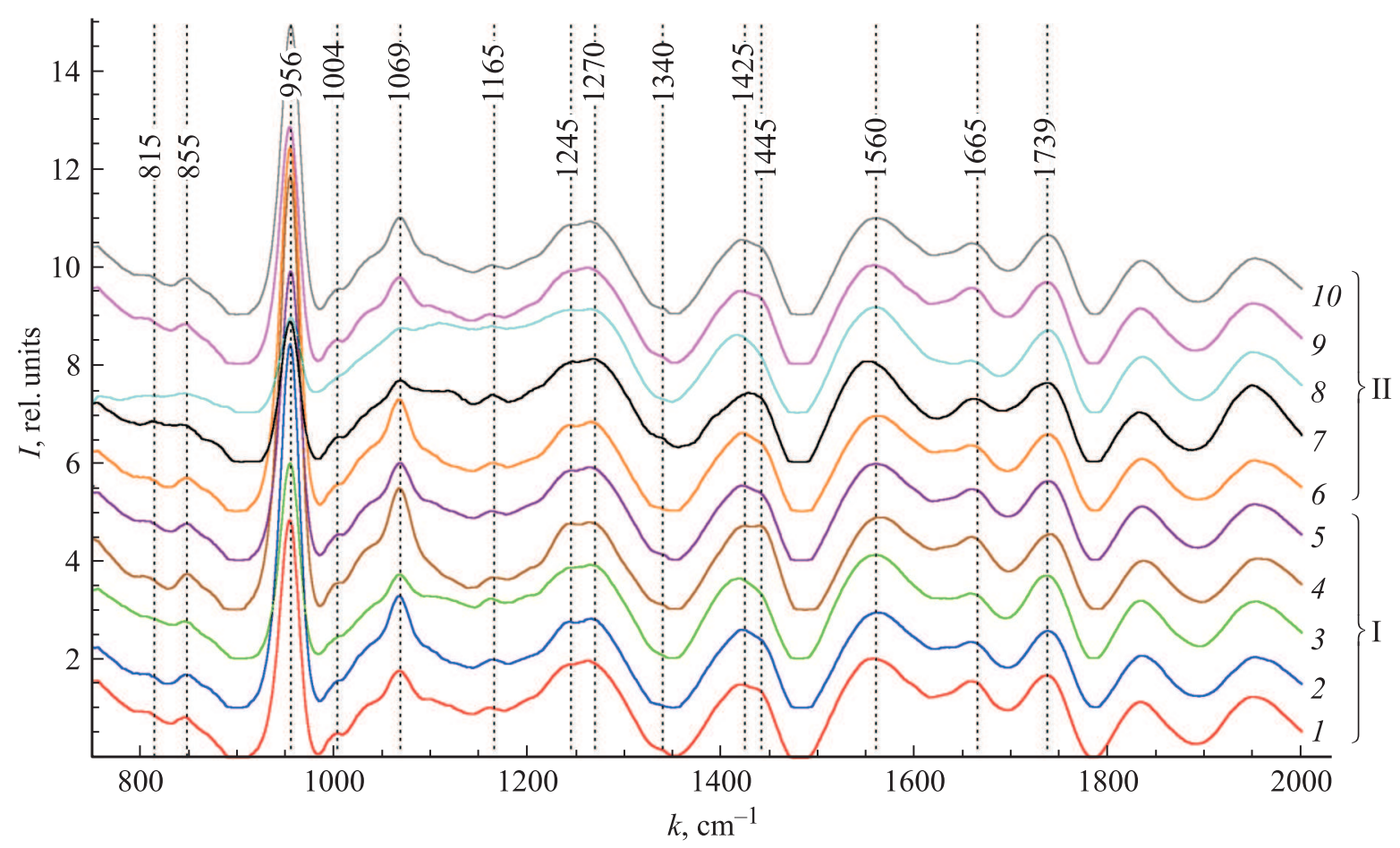

Рис. 3. Усредненные спектры кортикальных плечевых костей крыс. I - самки: 1 - контроль; 2 - вывешивание в течение 28 суток; 3 - вывешивание в течение 28 суток +глюкокортикоиды в дозе $40 \mathrm{mg} / \mathrm{kg}$ ежедневно; 4 - вывешивание в течение 28 суток + глюкокортикоиды в дозе $40 \mathrm{mg} / \mathrm{kg}$ ежедневно + ГАП в дозе $100 \mathrm{mg} / \mathrm{kg}$ однократно в первый день; $5-$ вывешивание в течение 28 суток + ГАП в дозе $100 \mathrm{mg} / \mathrm{kg}$ однократно в первый день. II - самцы: 6 - контроль; 7 - вывешивание в течение 28 суток; 8 - вывешивание в течение 28 суток + глюкокортикоиды в дозе $40 \mathrm{mg} / \mathrm{kg}$ ежедневно; 9 - вывешивание в течение 28 суток + глюкокортикоиды в дозе $40 \mathrm{mg} / \mathrm{kg}$ ежедневно + ГАП в дозе $100 \mathrm{mg} / \mathrm{kg}$ однократно в первый день; $10-$ вывешивание в течение 28 суток + ГАП в дозе $100 \mathrm{mg} / \mathrm{kg}$ однократно в первый день.

описывают линии КР, представленные на рис. 5 и имеющие наибольшее значение нормированного коэффициента канонической дискриминантной функции LD-1 по модулю. Чем выше значение LD-1 для переменной, тем в большей степени она влияет на наблюдаемую разницу в компонентном составе, что, например, видно из значения коэффициента $k_{956}$, соответствующего $\mathrm{P}$-О-симметричному валентному колебанию $\mathrm{PO}_{4}^{3-}\left(v_{1}\right)$ ГАП. Аналогично в костной ткани преобладают относительные интенсивности линий КР пролина, гидроксипролтина и фенилаланина 1000, 1031 и $1067 \mathrm{~cm}^{-1}$, соответствующих плоскостному валентному колебанию $\mathrm{C}-\mathrm{O}$ карбонат-иона ГАП $\mathrm{CO}_{3}^{2-}\left(v_{1}\right)$. Относительные интенсивности этих линий выше в образцах под действием глюкокортикоидов. Точность классификации составляет 77.7\%, 63.6\% перекрестно проверенных сгруппированных наблюдений классифицированы правильно.

На рис. 6 и 7 представлены основные различия в спектральном составе костной ткани животных различных полов. Положительные значения LD-1 характерны для группы костной ткани самцов. Для образцов костной ткани самок характерны более высокие относительные интенсивности линий ГАП 956, 1031 и $1067 \mathrm{~cm}^{-1}$, амида I $\left(k_{1659}\right)$ и линии $1448\left(\mathrm{CH}_{2}\right.$ изгиб и ножничные режимы коллагена и фосфолипидов). Для образцов костной ткани самцов преобладают относительные интенсивности линий амида III и амида II, 1130 (lipids), 1202 (phenilalanin), 1168 ( $(\mathrm{C}-\mathrm{O}-\mathrm{C})$, symmetricstretch), $1101 \mathrm{~cm}^{-1}\left(\mathrm{CO}_{3}^{2-}\left(v_{1}\right)\right.$, замещение $A$-типа). Точность классификации составляет 87.6\%, 77.7\% перекрестно проверенных сгруппированных наблюдений классифицированы правильно.

На рис. 8, 9 представлен анализ влияния однократной дозы $100 \mathrm{mg} / \mathrm{kg}$ ГАП. Основным отличием между группами является относительная интенсивность линии $956 \mathrm{~cm}^{-1}$, соответствующей фосфатной минеральной компоненте ГАП и преобладающей в группе образцов с влиянием однократной дозы ГАП. Различия на других спектральных линиях обусловлены другими факторами (пол, дозирование глюкокортикоидами, различие в вывешивании). Точность классификации составляет $86.0 \%$, $77.7 \%$ перекрестно проверенных сгруппированных наблюдений классифицированы правильно.

В итоге видно, что значения переменных $k_{856}, k_{878}$, $k_{956}, k_{1000}, k_{1031}, k_{1067}, k_{1448}, k_{1659}$ выше в образцах костной ткани самок с глюкокортикоидами в дозе $40 \mathrm{mg} / \mathrm{kg}$ ежедневно. Однократная доза ГАП тоже влияет на интенсивность линии ГАП, но только на линию $956 \mathrm{~cm}^{-1}$. Амплитуда линии КР амида І имеет обратную 


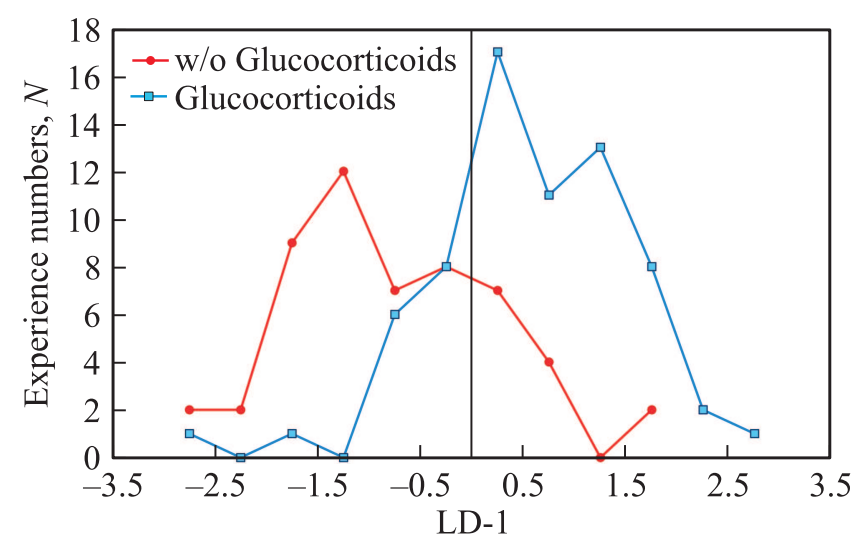

Рис. 4. Значения линейных дискриминантных функций для образцов костной ткани, разделение по признаку влияния глюкокортикоидов.

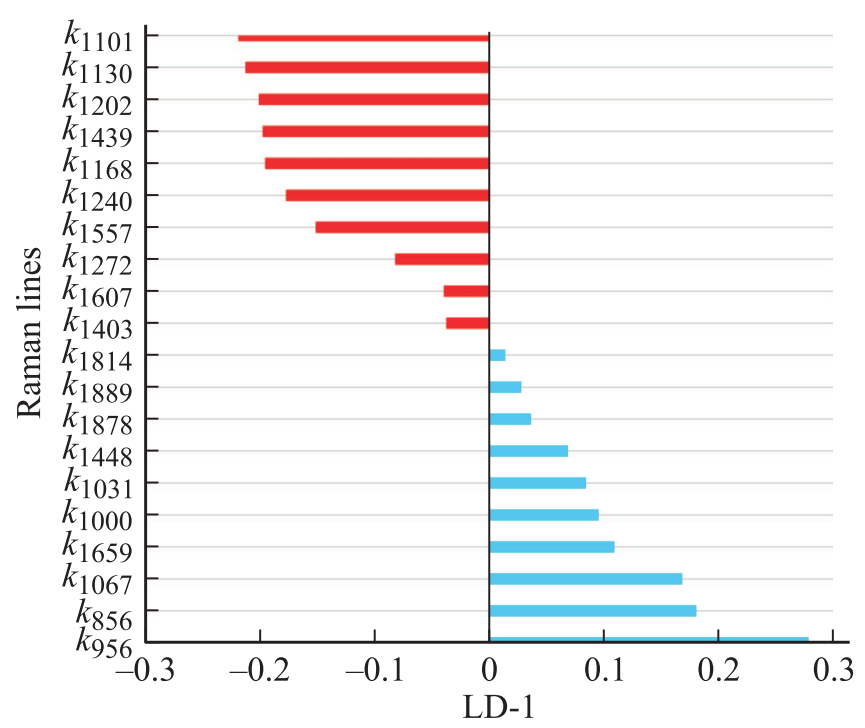

Рис. 5. Значения структурной матрицы для дифференциации образцов по признаку влияния глюкокортикоидов.

корреляцию с линиями амидов II и III в зависимости от пола и влияния глюкокортикоидов.

\section{Результаты биохимического анализа}

В ходе исследования не было выявлено гендерных различий в показаниях поверхностной микротвердости костной ткани и содержании $C$-концевых телопептидов сыворотки крови. Поэтому результаты представлены в виде средних по группам при объеме выборки $n=12$ в группе. Значения поверхностной микротвердости для бедренных костей снижены относительно контрольных в антиортостатической модели на $26 \%$ и в комбинированной - на $16 \%$. Введение ГАП оказывает особенно выраженный эффект в комбинированной модели: значения для данной группы выше значений аналогичной экспериментальной группы на 20\%. Аналогич-

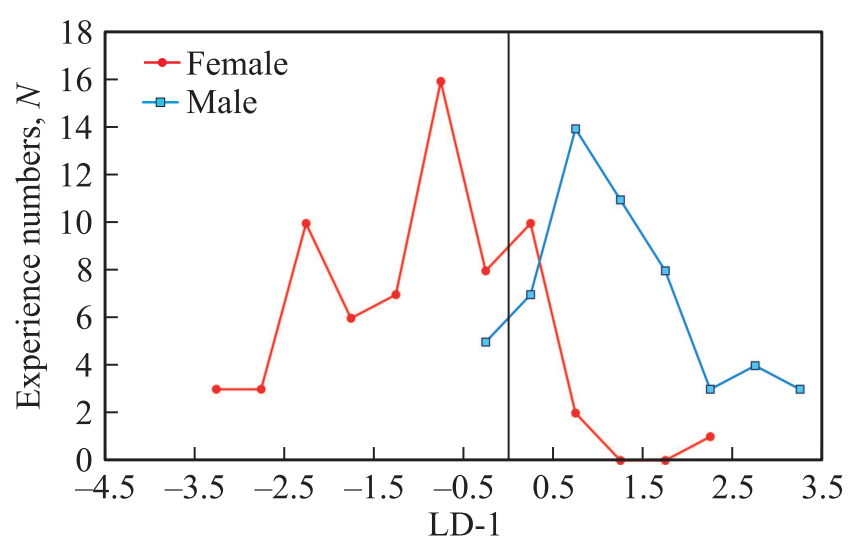

Pис. 6. Значения линейных дискриминантных функций для образцов костной ткани, разделение по полу животных.

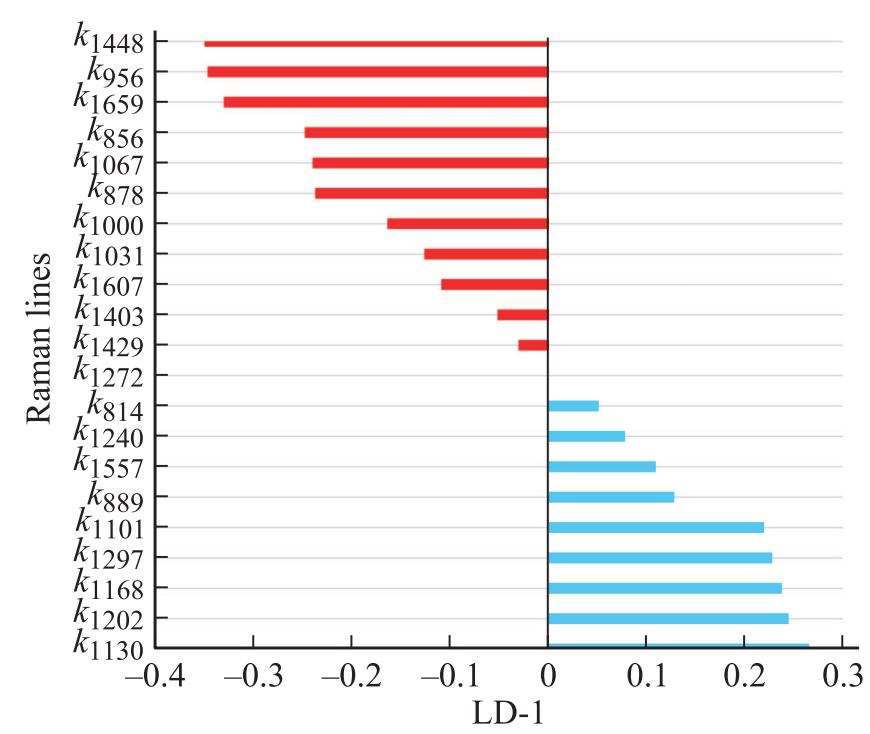

Рис. 7. Значения структурной матрицы для дифференциации образцов по признаку пола животных.

ная тенденция прослеживается и для тазовых костей. При введении ГАП в случае антиортостатического вывешивания поверхностная микротвердость костей таза увеличилась на $22 \%$ по сравнению с изолированной моделью антиортостатической остеорезорбции. В случае комбинированной модели с глюкокортикоидами (ГК) и вывешиванием при введении ГАП показатель для тазовых костей повысился на 25\%. Предположительно в результате перераспределения нагрузки на конечности происходит значительное ухудшение состояния ткани костей верхнего плечевого пояса: значения исследуемого параметра снижены относительно контрольных на 22 (плечо) и 3\% (лопатка) в антиортостатической модели, а комбинирование моделей резорбции снижает значения относительно контрольных для лопаточных костей на $29 \%$ относительно контроля и на $27 \%$ относительно простой антиортостатической модели. Инъекции ГАП улучшают состояние костной ткани как при изолиро- 


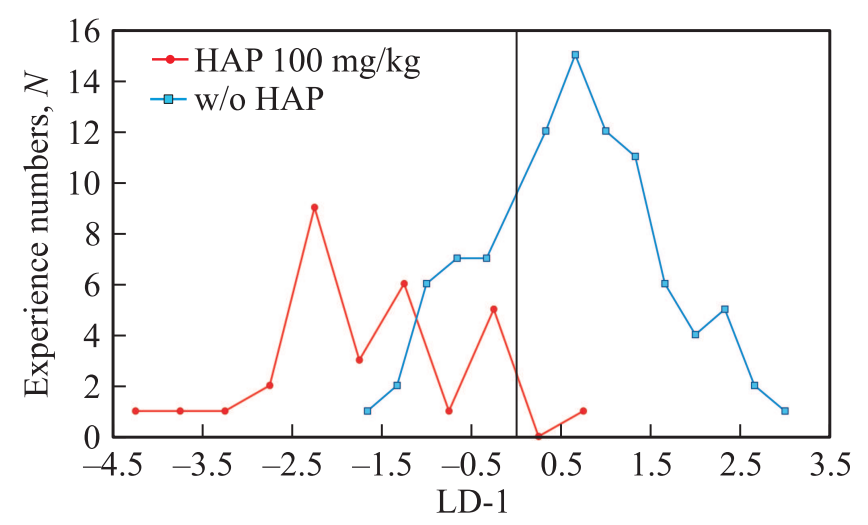

Рис. 8. Значения линейных дискриминантных функции для образцов костной ткани, разделение по признаку влияния однократной дозы ГАП.

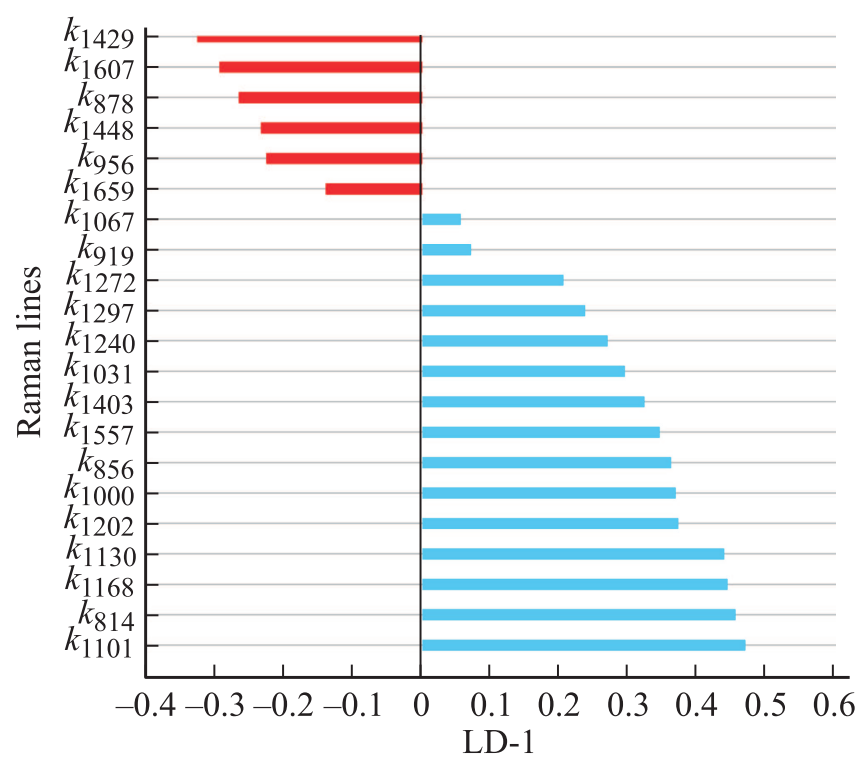

Pис. 9. Значения структурной матрицы для дифференциации образцов по признаку влияния однократной дозы ГАП.

ванном антиортостатическом вывешивании, так и при комбинированной модели: в комбинированной модели значения выше значений аналогичной экспериментальной группы на 49\% для лопаточных костей, на $12 \%$ для плечевых и на 3\% для нижнечелюстных. Аналогичные изменения отмечены и в группе с антиортостатическим вывешиванием и введением ГАП, а именно повышение на $16 \%$ для плечевых костей, на $10 \%$ для лопаточных и на 7\% для нижнечелюстных относительно результатов изолированного вывешивания (табл. 2).

Усиление костной резорбции у животных, участвовавших в антиортостатической модели, выражается увеличением концентрации $C$-концевых телопептидов на $43 \%$ (табл. 3). При дополнительной экзогенной нагрузке стероидами исследуемый показатель возрастает на 64\%. Введение ГАП на фоне совместного действия вывешивания и глюкокортикоидов приводит к повышению $\beta$-CrossLaps на $60 \%$, а при изолированном вывешивании на $39 \%$ по сравнению с контролем. Таким образом, измерение концентрации концевых телопептидов послужило подтверждением способности ГАП ослаблять процессы резорбции, вызванные действием глюкокортикоидов и изменением гравитации.

При всех заболеваниях скелета происходят нарушения процессов ремоделирования кости, что сопровождается возникновением отклонений в уровне биохимических маркеров [32]. C-концевые телопептиды представляют собой фрагменты коллагена I типа и являются наиболее информативными маркерами костной резорбции. При физиологически или патологически повышенной резорбции кости коллаген I типа разрушается быстрее, при этом соответственно повышается уровень фрагментов коллагена в крови [33]. Первичный остеопороз сопровождается отчетливым повышением уровня $C$-концевых телопептидов коллагена I типа [32]. Различают $\alpha$ - и $\beta$-изомеры CrossLaps. Доля $\beta$-изомеров возрастает при старении кости, поэтому повышение их концентрации свидетельствует о резорбции кости взрослого человека, а повышение уровня $\alpha$-изомеров - о резорбции молодой кости [3436]. Уровень $\beta$-CrossLaps в группах с моделированием глюкокортикоидной остеорезорбции повышается относительно контрольной группы. Инъекции ГАП экспериментальным животным нивелируют данные изменения.

\section{Результаты морфологического анализа}

На препаратах бедренных костей крыс после антиортостатического вывешивания и введения глюкокортикоидов в течение 28 суток по сравнению с препаратами животных интактной группы (рис. 10) отмечена резорбция костной ткани, апоптоз остеобластов, расширение межбалочного пространства, истончение и разволокнение костных балок (рис. 11). Выраженное

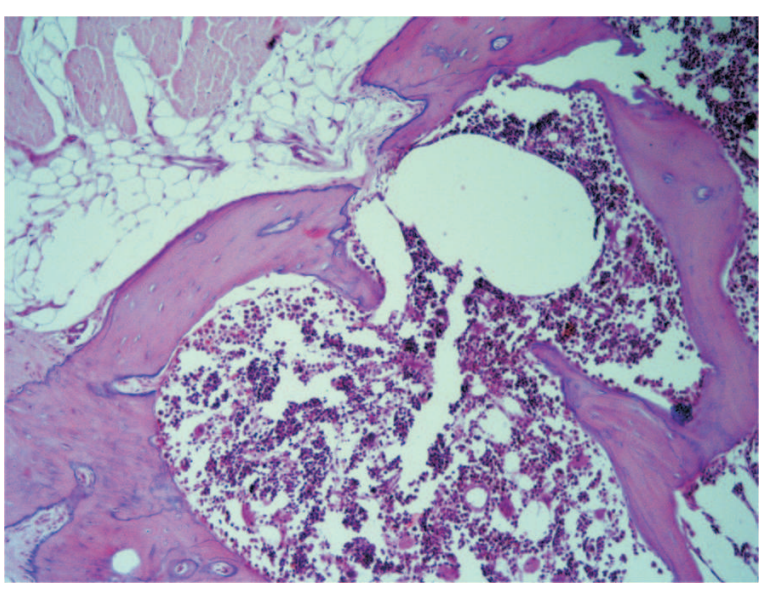

Рис. 10. Костная ткань интактных животных. Окраска: гематоксилин и эозин. Увеличение $20 \times 10$. 
Таблица 2. Значения поверхностной микротвердости костей крыс в комбинированной модели остеорезорбции, $\mathrm{kgs} / \mathrm{mm}{ }^{2}$

\begin{tabular}{l|c|c|c|c|c}
\hline \multicolumn{1}{c|}{ Группа } & Бедро & Таз & Плечо & Лопатка & Нижняя челюсть \\
\hline Контроль $(n=12)$ & $253.36 \pm 13.20$ & $199.06 \pm 8.95$ & $239.49 \pm 13.62$ & $234.82 \pm 8.17$ & $252.64 \pm 9.21$ \\
Вывешивание $(n=12)$ & $186.40 \pm 5.42^{\$}$ & $180.69 \pm 5.40$ & $186.50 \pm 8.26^{\#}$ & $226.72 \pm 9.69$ & $170.64 \pm 4.55^{\$}$ \\
Вывешивание + ГК $(n=12)$ & $211.64 \pm 5.71^{\#}$ & $173.83 \pm 4.73^{*}$ & $182.67 \pm 4.73^{\#}$ & $165.59 \pm 2.17^{\$}$ & $207.80 \pm 5.67^{\$}$ \\
Вывешивание + ГАП $(n=12)$ & $190.16 \pm 5.72^{\$}$ & $220.55 \pm 6.34$ & $216.26 \pm 5.45$ & $249.56 \pm 6.75$ & $182.63 \pm 5.53^{\$}$ \\
Вывешивание + ГК + ГАП $(n=12)$ & $254.87 \pm 9.93^{\&}$ & $216.65 \pm 6.52^{\&}$ & $205.42 \pm 5.94^{*}$ & $246.11 \pm 10.65^{\&}$ & $213.85 \pm 9.90^{\#}$
\end{tabular}

Примечание. ${ }^{*}$ - отличия от контроля статистически достоверны с уровнем значимости $p<0.05$; \# достоверны с уровнем значимости $p<0.01 ; \$$ - отличия от контроля статистически достоверны с уровнем значимости $p<0.001 ;$ \& - отличия от группы Вывешивание + ГК статистически достоверны с уровнем значимости $p<0.01{ }^{£}$ - отличия от группы Вывешивание статистически достоверны с уровнем значимости $p<0.05$.

Таблица 3. Концентрация $\beta$-CrossLaps в сыворотке крови крыс в комбинированной модели остеорезорбции, $\mathrm{pg} / \mathrm{ml}$

\begin{tabular}{c|c|c|c|c}
\hline \multicolumn{5}{c}{ Группы животных } \\
\hline $\begin{array}{c}\text { Контроль } \\
(n=12)\end{array}$ & $\begin{array}{c}\text { Вывешивание } \\
(n=12)\end{array}$ & $\begin{array}{c}\text { Вывешивание }+\Gamma \text { ГК } \\
(n=12)\end{array}$ & $\begin{array}{c}\text { Вывешивание }+ \text { ГАП } \\
(n=12)\end{array}$ & $\begin{array}{c}\text { Вывешивание }+ \text { ГК }+ \text { ГАП } \\
(n=12)\end{array}$ \\
\hline $69.33 \pm 1.82$ & $99.21 \pm 2.13^{\$ \ddagger}$ & $113.73 \pm 3.62^{\$}$ & $96.01 \pm 1.12^{\$ \ddagger}$ & $111.08 \pm 3.51^{\$}$
\end{tabular}

Примечание. ${ }^{\S}$ — отличия от контроля статистически достоверны с уровнем значимости $p<0.001$; $—$ отличия от группы с ГК статистически достоверны с уровнем значимости $p<0.01$.

истончение костных трабекул наблюдали в костной ткани крыс после антиортостатического вывешивания в течение 28 суток (рис. 12). После антиортостатического вывешивания и введения ГАП в дозе $100 \mathrm{mg} / \mathrm{kg}$ наблюдается умеренное истончение костных трабекул, костные балки с активными остеобластами (рис. 13). После антиортостатического вывешивания, введения глюкокортикоидов, введения ГАП в дозе $100 \mathrm{mg} / \mathrm{kg}$ отмечено слабое снижение объема части трабекул, незначительное уменьшение толщины балок и ширины межбалочного пространства (рис. 14).

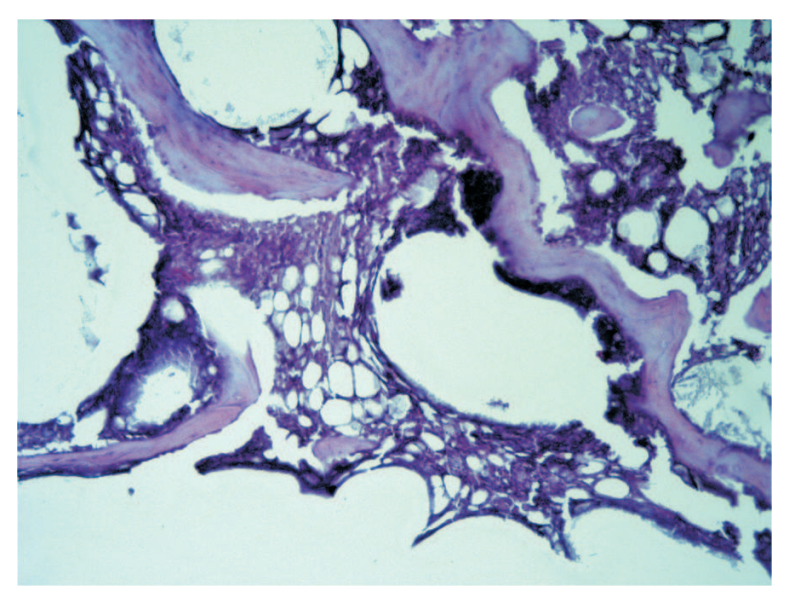

Рис. 11. Костная ткань крыс после антиортостатического вывешивания и введения глюкокортикоидов $40 \mathrm{mg} / \mathrm{kg} 28$ суток (резорбция костной ткани, апоптоз остеобластов, расширение межбалочного пространства, истончение и разволокнение костных балок).Окраска: гематоксилин и эозин. Увеличение $20 \times 10$.

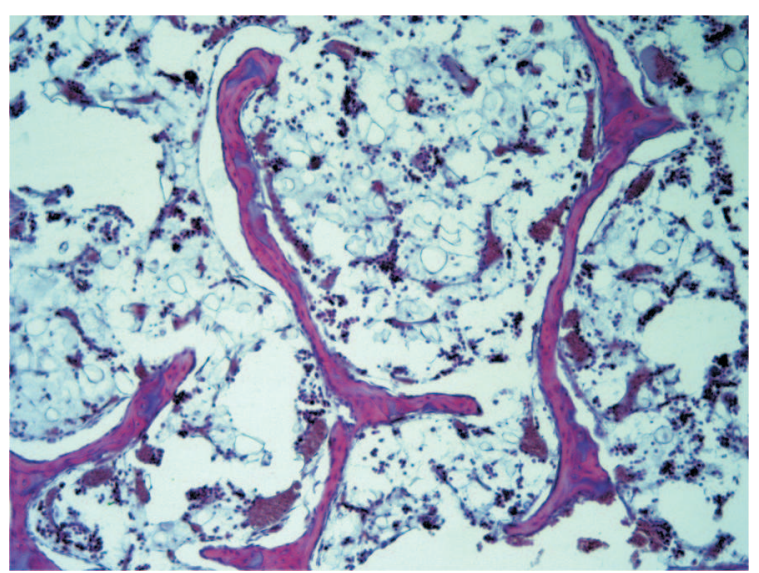

Рис. 12. Костная ткань крыс после антиортостатического вывешивания 28 суток (выраженное истончение костных трабекул,). Окраска: гематоксилин и эозин. Увеличение $20 \times 10$.

\section{Заключение}

В результате проведена деконволюция спектров методом подбора спектрального контура и деконволюции функции Гаусса в программной среде MagicPlotPro 2.7.2., что позволило провести расширенный компонентный качественный и количественный анализ костной ткани у крыс в условиях микрогравитации. Установлены основные спектральные различия исследуемых объектов (контрольные образцы, образцы с моделью остеопороза, образцы с моделью остеопороза при лечении ГАП), наблюдающиеся на линиях 855, 956, $1069 \mathrm{~cm}^{-1}$. Полученные результаты впоследствии могут быть применены 


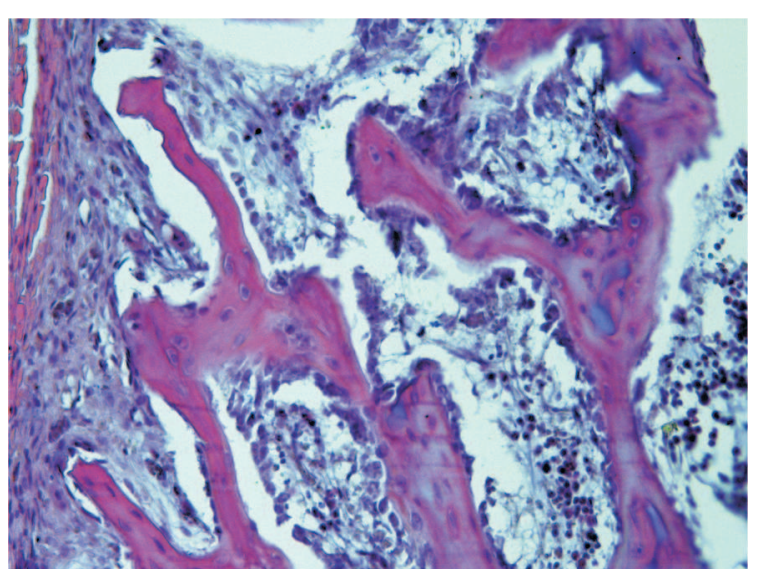

Рис. 13. Костная ткань крыс после антиортостатического вывешивания 28 суток и введения гидроксиапатита в дозе $100 \mathrm{mg} / \mathrm{kg}$ (умеренное истончение костных трабекул, костные балки с остеобластами). Окраска: гематоксилин и эозин. Увеличение $20 \times 10$.

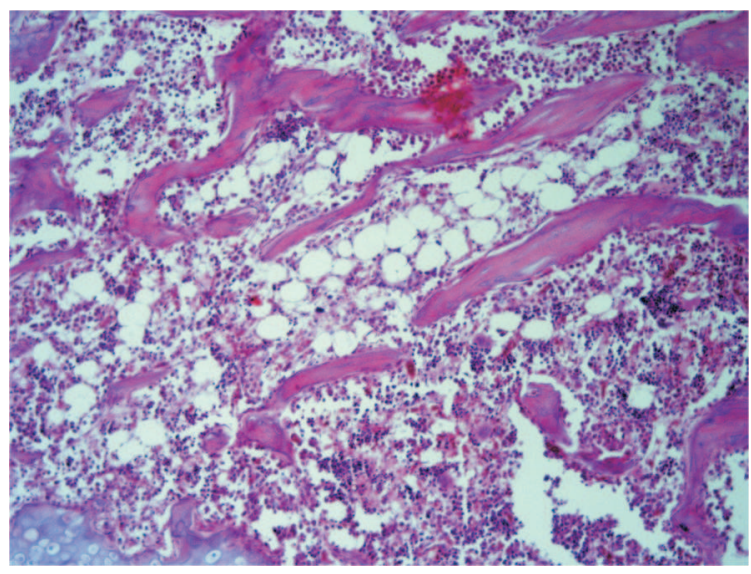

Рис. 14. Костная ткань крыс после антиортостатического вывешивания, введения глюкокортикоидов $40 \mathrm{mg} / \mathrm{kg} 28$ суток и введения ГАП в дозе $100 \mathrm{mg} / \mathrm{kg}$ (слабое снижение объема части трабекул, незначительное уменьшение толщины балок и ширины межбалочного пространства). Окраска: гематоксилин и эозин. Увеличение $20 \times 10$.

для профилактики и лечения остеопороза у космонавтов, поддержания здоровья экипажей во время длительных межпланетных миссий, а также для коррекции нарушений минерального гомеостаза и других заболеваний, связанных с нарушением костно-хрящевого метаболизма и минерального гомеостаза.

Установлено, что поверхностная микротвердость бедренных костей крыс в условиях антиортостатической разгрузки снижается на $26 \%$, а в комбинированной с глюкокортикоидами - на $16 \%$, что свидетельствует о снижении устойчивости костной ткани к микроповреждениям. Инъекции ГАП ослабляют этот эффект: значения поверхностной микротвердости повышаются при введении ГАП на фоне как комбинированной модели резорбции, так и при изолированном антиортостатическом вывешивании.

Концентрация концевых телопептидов коллагена в сыворотке крови крыс возрастает по сравнению с контрольными значениями в условиях микрогравитации на 35-43\%. Внутримышечное введение лабораторным животным аллогенного ГАП на фоне стероидиндуцированной остеорезорбции и изменения гравитации приводит к нормализации состояния костной ткани.

Использованный комплекс методов позволил установить, что внутримышечное введение лабораторным животным аллогенного ГАП в условиях измененной гравитации приводит к нормализации структурно-функционального состояния костной ткани.

\section{Соблюдение этических стандартов}

Все применимые международные, национальные и/или институциональные принципы ухода и использования животных были соблюдены.

\section{Финансирование работы}

Исследования проводились при финансовой поддержке РФФИ, проект № 18-315-20017-18.

\section{Конфликт интересов}

Авторы заявляют, что у них нет конфликта интересов.

\section{Список литературы}

[1] Привалов В.Е., Шеманин В.Г. // Опт. и спектр. 1997. Т. 82. № 4. C. 700.

[2] Привалов В.Е., Шеманин В.Г. // Опт. и спектр. 1997. Т. 82. № 5. C. 873 .

[3] Меркурьев С.В., Привалов В.Е., Шеманин В.Г. // Письма ЖТФ. 2000. Т. 26. № 1. C. 45.

[4] Arai F., Miyamoto T., Ohneda O. // J. Exp. Med. 1999. V. 190. P. 1741.

[5] Hildebrand T., Ruegsegger P. // Comput. Methods Biomech. Biomed. Engin. 1997. N 1. P. 15.

[6] Простяков И.В. Исследование изменений минеральной плотности и структурной организации костной ткани после воздействия факторов космического полета и при их наземном моделировании. [Электронный ресурс] Режим доступа: https://www.dissercat.com/content/ issledovanie-izmenenii-mineralnoi-plotnosti-istrukturnoi-organizatsii-kostnoi-tkani-posle-v

[7] Caillot-Augusseau A., Vico L., Heer M., Voroviev D., Souberbielle J.C. et al. // Clin. Chem. 2000. V. 46. P. 1136-1143.

[8] Clowes J.A., Riggs B.L., Khosla S. // Immunol. Rev. 2005. V. 208. P. 207.

[9] Егоров А.Д. Теория и методологии медицинского контроля в длительных космических полетах. [Электронный pecypc] Режим доступа: http://www.imbp.ru/ webpages/win1251/science/Egorov_actsp.html 
[10] Huntoon L., Grigoriev C.S. // Amer. Astronaut. Soc. Publ. 1998. V. 94. P. 220.

[11] Smith S.M., Wastney M.E., O'Brien K.O., Morukov B.V., Larina I.M. et al. // J. Bone Miner. Res. 2005. V. 20. P. $208-218$.

[12] Macias B.R., Swift J.M., Nilsson M.I., Hogan H.A., Bouse S.D., Bloomfield S.A. // J. Appl. Physiol. 2012. V. 112. P. 918-925.

[13] Писарева Е.В., Соколовская А.Б., Власов М.Ю. // Вестник СамГУ. 2012. № 3/2(94). С. 37-44.

[14] Halloran B.P., Bikle D.D., Cone C.M., Morey-Holton E. // Am. J. Physiol. 1988. V. 255. P. E875-E879.

[15] Рубин М.П. // Радиология-практика. 2009. Т. 3. С. 12.

[16] Пьявченко Г.А., Алексеев А.Г., Воробьёв Е.В. // Биомед. фотоника. 2018. Т. 15. № 5. С. 37.

[17] Ильин Е.А., Новиков В.Е. // Косм. биол. и авиакосм. мед. 1980. T. 14. № 3. С. $79-80$.

[18] Timchenko P.E., Timchenko E.V., Dolgushkin D.A., Volova L.T., Markova M.D. // J. Opt. Technol. 2017. V. 84. N 6. P. 423.

[19] Timchenko P.E., Timchenko E.V., Volova L.T. // J. Phys. Conf. Ser. 2018. V. 1038. N 1.

[20] Тимченко П.Е., Тимченко Е.В., Волова Л.Т., Фролов О.О. // Опт. спектр. 2019. Т. 126. N 6. С. 843; Timchenko P.E., Timchenko E.V., Volova L.T., Frolov O.O. // Opt. Spectrosc. 2019. V. 126. N 6. P. 769.

[21] Timchenko P.E., Timchenko E.V., Volova L.T., Nosova M.A., Frolov O.O., Kiyko N.K., Volov N.V. // Opt. Memory and Neural Networks. 2018. V. 27. N 1. P. 46.

[22] Christenson R.H. // Clinical Biochemistry. 1997. V. 30. N 8. P. 573.

[23] Fonseca H. // Sports Med. Rev. 2014. V. 44. N 1. P. 37. doi 10.1007/s40279-013-0100-7 2013

[24] Morris M.D. // Sports Med. 2011. V. 459. N 8. P. 2160. doi 10.1007/s11999-010-1692

[25] Boivin G. // Sports Med. 2013. V. 24. N 8. P. 2153. doi 10.1007/s00198-012-2228

[26] Połomska M., Kubisz L., Kalawski R., Oszkinis G., Filipiak R., Mazurek A. // Acoust. Biomed. Engine. 2019. V. 17. N 1. P. 24

[27] Tarnowski C.P., Ignelzi Jr M.A., Morris M.D. // J. Bone Miner. Res. 2002. V. 17. N 6. P. 1118.

[28] Movasaghi Z., Rehman S., Rehman I.U. // Appl. Spectr. Rev. 2007. V. 42. N 5. P. 493.

[29] Martin Lee. Imaging intra-cellular wear debris with coherent anti-Stokes. [Электронный ресурс] Режим доступа: https://era.ed.ac.uk/handle/1842/7735

[30] Rajesh Kumar, Gajendra P. Singh, Kirsten M. Gronhaug, Nils K. Afseth, Catharinade Lange Davies, Jon O. Drogset, Magnus B. Lilledahl // Int. J. Mol. Sci. 2015. V. 16. P. 9341. doi 10.3390/ijms16059341

[31] Mekhala Raghavan. Investigation of Mineral and Collagen Organization in Bone Using Raman Spectroscopy. [Электронный ресурс] Режим доступа: https://deepblue.lib.umich.edu/handle/2027.42/84443

[32] Ihtesham U. // Appl. Spectr. Rev. 2007. V. 42. N 5. P. 493.

[33] Sukhoduba L.F., Moseke C., Sukhodub L.B., SulkioCleff B., Maleev V.Ya., Semenov M.A., Bereznyak E.G., Bolbukh T.V. // J. Mol. Struct. 2004. V. 704. P. 53. doi 10.1016/j.molstruc.2003.12.061

[34] Рытикова Н.С., Смиронова М.А. // Журнал международной медицины. 2016. Т. 21. № 4. С. 32.
[35] Коровина Н.А., Свинцищкая В.И. // Педиатрия. 2010. Т. 89. № 6. C. 12.

[36] Дрыгина Л.Б., Трофимова И.В., Саблин О.А., Никифорова А.М. Современные методы диагностики, профилактики и лечения остеопороза. / Учебное пособие. СПб: ВЦЭРМ им. А.М. Никифорова МЧС России, 2011. 86 с. 